\title{
Are watches in the ambulances and the Emergency Department synchronized?
}

\author{
Mikkel Brabrand $^{1 *}$, Susanne Hosbond ${ }^{1}$, Dan Brun Petersen ${ }^{2}$, Lars Folkestad ${ }^{1}$ \\ From Danish Society for Emergency Medicine: Research Symposium 2010 \\ Roskilde, Denmark. 20-21 May 2010
}

\section{Background}

Prognostication in patients with cardiac arrest (CA) is (amongst other things) dependent on the duration of the CA. However in patients with out-of-hospital CA, the patient is often handed over from the ambulance crew to hospital staff. During the hand-over ambulance crews often present a printed summary of events during the resuscitation attempt from the defibrillator.

As treatment for the CA is started in the prehospital phase, the hospital staff needs reliable information on the duration of the CA in order to prognosticate the patient properly. It is therefore important that the ambulance crew and the hospital staff use watches that are synchronized. Unsynchronized watches can lead to both over and underestimation of the duration of the CA.

The aim of our study was to examine if the watches in defibrillators in ambulances were synchronized with the wall mounted clocks at Emergency Departments (ED).

\section{Methods}

Using a laptop computer with a wireless connection to the internet, we synchronized the watch with an atomic clock (http://time.gov). We then compared the reading of this clock with the wall mounted clocks in the rooms most often used for resuscitation at the Emergency Departments at Sydvestjysk Sygehus Esbjerg and Kolding Sygehus. We also compared the watches in the defibrillators of a convenience sample of ambulances in both Esbjerg and Kolding with the atomic clock.

Deviation from the atomic clock was calculated in seconds and is presented as median (IQR).

\section{Results}

There were two watches in the resuscitation rooms in the ED in Kolding, both were exactly on time. We examined the defibrillator in seven ambulances in Kolding and found a median deviation of 45 seconds (1102). In Esbjerg the watches in the resuscitation rooms in the ED were a median $150(120-180)$ seconds ahead of the atomic clock. In the ambulances in Esbjerg the defibrillators were a median $252(97-315.5)$ seconds ahead.

\section{Conclusion}

We found a maximum deviation between the watches in defibrillators in ambulances and wall mounted clocks in the ED's of approx. 7.5 minutes. However most deviations were minor and would probably have no significant impact on prognostication of the individual patient.

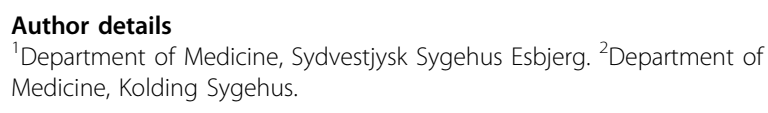

Published: 17 September 2010

doi:10.1186/1757-7241-18-S1-P3

Cite this article as: Brabrand et al: Are watches in the ambulances and the Emergency Department synchronized? Scandinavian Journal of Trauma, Resuscitation and Emergency Medicine 2010 18(Suppl 1):P3.

* Correspondence: mikkel@brabrand.net

'Department of Medicine, Sydvestjysk Sygehus Esbjerg

Full list of author information is available at the end of the article 